\title{
Schreiben am Limit
}

\section{Wissenschaftsjournalismus zwischen Anspruch und Wirklichkeit}

\author{
Rainer Kurlemann
}

Ein Wissenschaftsjournalist kommt bei einer Party schnell ins Gespräch spätestens, wenn er seinen Beruf erwähnt. Ihm schlägt eine positiv gefärbte Mischung aus Respekt (Chemie? - das habe ich in der Schule nie verstanden) und Neugierde (das muss ja sehr spannend sein) entgegen. Wissenschaftsjournalist könnte ein Traumberuf sein, aber das ist heute leider nicht mehr der Fall. Denn zwischen Anspruch und Wirklichkeit klaffen Welten.

Wenn man den Anspruch beschreiben will, der an den Wissenschaftsjournalismus und an seine Autoren gestellt wird, müssen mindestens vier verschiedene Perspektiven berücksichtigt werden. Leider sind diese Blickwinkel nur in Ausnahmefällen deckungsgleich und ein freier Journalist wie ich muss sich Gedanken machen, wie er das Problem der unterschiedlichen Erwartungshaltungen im Alltag handhaben will.

Die erste Perspektive entscheidet maßgeblich über den wirtschaftlichen Erfolg eines freiberuflichen Journalisten. Als Unternehmer ist er derzeit zumeist - noch - abhängig von den Redaktionen und Verlagen, die nicht immer den Fähigkeiten des Autors blind vertrauen, sondern in unterschiedlichem Ausmaß Vorgaben setzen. Manchmal gibt es diese Vorgaben schon vor der Auftragsvergabe, wenn Redaktionen beispielsweise ein Exposé verlangen, das im Extremfall nicht nur das Thema (ausführlich) erklärt, sondern auch die Ansprechpartner und ein paar Thesen des Textes vorab auflistet. Seriöse Journalisten können ein solches Exposé nur dann erstellen, wenn sie sich sicher sein können, dass die Recherche diese Ergebnisse liefern

\footnotetext{
R. Kurlemann $(\bowtie)$

Düsseldorf, Deutschland
} 
wird. Natürlich ist das manchmal der Fall, doch weitaus spannender ist die offene Recherche zu den Entwicklungen in einem Forschungsgebiet, deren Verlauf sich erst aus den Interviews mit den Beteiligten ergibt.

Als Journalist kann man von Glück sagen, wenn man mit einer Redaktion zusammenarbeiten darf, die sich mit der Ankündigung zufriedengibt, dass man einen Fachkongress, einen namhaften Forscher oder ein spezielles Projekt besuchen und von dort eine spannende Geschichte mitbringen wird, die sich mit weiterer Recherche zu einem lesenswerten Artikel abrunden lässt. Dieses Vorgehen verlangt vom Autor eine gute Kenntnis, welche Themen schon hinreichend behandelt wurden, damit die Redaktion etwas Neues bekommt. Und es benötigt den festen Willen, bei der Themensuche nicht immer dem Licht der Scheinwerfer zu folgen, sondern nach der weniger bekannten, aber zukunftsgerichteten Forschung zu suchen. Wenn diese beiden Bedingungen erfüllt sind, sich eine Portion Glück einstellt, gekoppelt mit der Bereitschaft, auch das Scheitern zu riskieren, dann sind die Voraussetzungen geschaffen, dass Redaktion und Journalist gleichermaßen Spaß an der Arbeit und dem Ergebnis haben.

Eine andere Vorgabe, die Redaktionen und Verlage gern machen, betrifft die Textkategorie: Soll es ein Interview sein, eine Reportage, ein Feature oder eine Analyse, vielleicht im Frage-Antwort-Stil? Soll der nachrichtliche Teil eher beiläufig berichtet werden oder den Kern des Textes bilden? Gibt es eine Hauptperson, deren Arbeit oder Schicksal so typisch ist, dass es gerechtfertigt erscheint, das ganze Thema daran aufzuhängen? Oder wird der Text eher den Widerspruch zwischen verschiedenen Positionen darstellen und seine Qualität auch daraus gewinnen, dass der Autor ein Thema seit Jahren bearbeitet, seine Entwicklung kennt und viele Facetten beleuchtet? Diese Fragen sind oft hilfreich und angenehm. Gute Texte bleiben das A und O des Journalismus. Gerade schwierige Themen wird der Leser nur dann lesen, wenn er sie attraktiv dargeboten bekommt. Die kleine Gruppe der Technikund Wissenschaftsenthusiasten, die alles lesen, was ihnen angeboten wird, reicht als Zielgruppe für die wichtigen Fragestellungen, die aktuell in der Wissenschaft entstehen, nicht aus. Ein Freiberufler weiß im Regelfall bereits, warum er sein Thema genau dieser Zeitung oder genau diesem Magazin angeboten hat. Er kennt Vorlieben und Vergleichbares, macht Vorschläge, welche Textkategorie zum Thema passen könnte.

Wenn ich aber gefragt werde, ob ich nicht noch gleichzeitig ein Video liefern könne, lehne ich ab. Ich bin schreibender Journalist und glaube nicht an das Konzept der Eier legenden Wollmilchsau als kostensparendes Universaltalent. Doch manche Verlage haben diesen Gedanken noch nicht aufgegeben. Früher hörte man außerdem noch einen Hinweis, ob der Text „online“ oder 
„print" erscheinen wird, verbunden mit der Bitte, den Text entsprechend anzupassen. Glücklicherweise verschwindet diese Denkweise in den Redaktionen. Print und Online verlangen und ermöglichen zwar eine unterschiedliche Präsentation des gleichen Themas. Aber aus meiner Sicht gibt es keine Print- oder Online-Texte, sondern nur gute oder schlechte Texte.

Damit haben wir die zweite Perspektive für den Anspruch eines Textes erreicht, nämlich den Blickwinkel der Leser oder der Nutzer. Redaktionen verweisen gern darauf, dass die Leser nach ihrer Ansicht bestimmte Dinge von einem Text erwarten und Vorlieben entwickelt haben, die es zu berücksichtigen gilt. Das betrifft häufig den Aufbau eines Textes, wie ein gelungener Einstieg sein sollte und was als zweiter Absatz folgen muss. Es ist selbstverständlich, dass Journalisten sich für das Interesse der Leser interessieren müssen. Aber ich bin mir manchmal nicht sicher, ob alle Redaktionen ihr Wissen über die Leserwünsche tatsächlich mit der gleichen Sicherheit gewonnen haben, wie sie es von den Wissenschaftlern, über die sie schreiben, verlangen würden. Aber das ist ein anderes Thema und führt zu weit weg vom Anliegen dieses Buches. Interessant ist jedoch, dass bei der Vergabe von Journalistenpreisen häufig diejenigen ausgezeichnet werden, die etwas Innovatives und Neues entwickelt haben, und nur selten diejenigen, die etwas Bewährtes und im positiven Sinne Berechenbares anbieten.

Als Freiberufler bedauere ich es, dass viele Verlage die Informationen über die Leserschaft häufig wie ein Geschäftsgeheimnis hüten. Das ist einer der Gründe, warum ich mich für eine Mitgliedschaft bei den RiffReportern (vgl. den Beitrag von Christian Schwägerl) entschieden habe. Bei diesem innovativen Projekt entfallen nicht nur manche - seien wir ehrlich - zuweilen für alle Beteiligten mühsamen Diskussionen über die Umsetzung von Themenvorschlägen, sondern öffnet sich gleichzeitig die Chance für einen intensiveren Kontakt zu den Lesern, der für Journalisten sehr wichtig ist. Journalisten können auch direkt mit ihnen in Kontakt treten, wenn sie sich nicht nur auf das Schreiben von Artikeln beschränken, sondern ihr Wissen in der Öffentlichkeit präsentieren, auf Lesungen, in Bibliotheken oder bei Vorträgen beispielsweise.

Die dritte Perspektive ergibt sich aus dem Blick der Wissenschaftler auf die Arbeit eines Journalisten. Die Ansprüche der Forscher sind dabei durchaus unterschiedlich. Einige Wissenschaftler haben mir nach der Veröffentlichung des Textes gesagt, dass ich ihre Arbeit besser erklären könne als sie selbst. Andere Forscher erwähnen schon während des Interviews, dass sie sich für den Text nicht interessieren und ihn nicht lesen werden. Wissenschaftler, die Erfahrung als Experten in der Politikberatung besitzen, haben andere Erwartungen an die Vertreter der Medien als Forscher, die vielleicht zum ersten Mal einen Journalisten treffen und sich freuen, dass ihr 
Anliegen endlich einmal größer präsentiert wird. Journalisten sollten sich zudem dafür interessieren, über welche Quellen ihre Gesprächspartner sich finanzieren. Es gibt noch immer viele Idealisten unter den Forschern und auch solche, die ihr Auskommen gesichert haben. Aber manchmal müssen Wissenschaftler auch Geld sammeln und da kann auch ein Bericht in einer Zeitung oder in einem Magazin hilfreich sein. So unterschiedlich diese Rahmenbedingungen sind, so unterschiedlich verlaufen auch die Gespräche mit den Experten. Doch ein Wissenschaftsjournalist sollte nie danach streben, die Erwartungen der Forscher zu erfüllen. Er sollte sein Gegenüber aber fair behandeln (der Begriff ist hier nicht im Sinne von schonend gemeint), vor allem, wenn er regelmäßig über ein Forschungsgebiet berichten will.

Über das Verhältnis zwischen Journalist und Wissenschaftler ist viel gesprochen worden. Ich erinnere mich an eine Diskussion, ob Wissenschaftsjournalismus überhaupt notwendig sei. Einige Kollegen in der Redaktion vertraten damals die Auffassung, dass alle Journalisten über die Fähigkeit verfügen, komplizierte Sachverhalte darzustellen, wenn sie nur genügend Zeit bekommen, um Fragen zu stellen. Deshalb sei der Wissenschaftsjournalist als spezieller Fachkollege nicht nötig. Vor diesem Hintergrund werden feste Stellen für Wissenschaftsredakteure gern umgewidmet. Dabei nährt sich dieser Zweifel aus einem Missverständnis: nämlich dass der Kern der Arbeit von Wissenschaftsjournalisten vergleichbar mit der Tätigkeit eines Übersetzers sei. Es gehe darum, die Forschungsergebnisse für jedermann verständlich zu machen. Folgt man diesem Gedanken, dann wird die Sendung mit der Maus zur besten Form des Journalismus, denn Kinder sind wohl die schwierigste Zielgruppe für wissenschaftliche Inhalte.

In meinem Alltag nimmt die Rolle des Übersetzers aber immer weniger Platz ein. Gute Wissenschaftsjournalisten sind kritische Beobachter, als solche werden sie auch von den Redaktionen geschätzt. Sie lernen im Laufe der Jahre, das wirklich Erreichte von dem zu trennen, was Forscher nur ankündigen oder erhoffen. Gerade zu Beginn von millionenschweren Forschungsprogrammen passen Ankündigung und Wirklichkeit oft nicht zusammen. Es gehört zur Natur der Wissenschaft, dass die Wissenschaftler die Ergebnisse ihrer Forschung nicht kennen. Aber leider gehört es zur heutigen Zeit, dass neue technische Möglichkeiten angekündigt werden, die weit von dem entfernt sind, was realistisch ist. Viele Journalisten nehmen das auf, lassen der Fantasie bereitwillig freien Lauf oder räumen ihr zumindest mehr Platz ein als gerechtfertigt. Dadurch gleiten manche Debatten über wissenschaftliche Themen in ein Fahrwasser, dem sie nicht mehr entkommen können, das aber ihrer Bedeutung nicht entspricht. Beispiele dafür sind Siedlungen auf dem Mars oder die Produktion von Designer-Babys, die nach 
dem Stand der Wissenschaft beide ins Reich der Träume gehören und Jahrzehnte entfernt sind.

Viele Kollegen, die sich in der Wissenschaft nicht auskennen, schreiben trotzdem über solche Entwicklungen, als seien die genannten Beispiele in einer naheliegenden Zukunft Realität. In anderen Ressorts kommt das seltener vor. Stellen wir uns vor, ein Journalist würde über den möglichen Wegfall der nationalen Grenzen berichten, durch die ein einziger Staat entsteht: das gemeinsame Europa. Dieses Szenario ist denkbar, aber der Aufschrei unter den Politikjournalisten nach dem Erscheinen eines solchen Textes wäre groß, denn die Wahrscheinlichkeit für das grenzenlose Europa ist (derzeit) gering. Wissenschaftsjournalisten müssen dagegen damit leben, dass ihre Themen teilweise so behandelt werden, als ob sie schon morgen für den Alltag relevant seien. Aus diesem Dilemma ergibt sich der Anspruch, den Wissenschaftsjournalisten an die Gespräche mit Forschern stellen müssen: Die fachgerechte Einordnung von Ankündigungen und Ergebnissen aus der Wissenschaft hat einen mindestens so großen Stellenwert wie die bloße Nachricht.

Damit habe ich längst die vierte Perspektive für den Anspruch an einen Wissenschaftsjournalisten erreicht: Es ist der Anspruch, den jeder einzelne Journalist an seine Arbeit stellt. Neben den klassischen Ansprüchen an die Arbeit von Journalisten kennen Wissenschaftsjournalisten auch eine besondere Herausforderung: Sie müssen Nachrichten und Ergebnisse vermitteln, deren Bedeutung den meisten Lesern fremd ist.

Und wer das richtige Selbstverständnis für Journalismus hat, wird darin seine größte Motivation für die Ausübung dieses Berufs sehen. Ein Beispiel: Der Anteil der Bevölkerung, der weiß, was sich hinter der Genschere CRISPR/Cas verbirgt, ist ohnehin schon sehr niedrig. Wenn Genetiker dieses Werkzeug nun einsetzen, um Babys mit verändertem Erbgut zu erzeugen, begibt sich der Leser quasi auf eine Reise in eine Art Terra incognita. Der erste Reisebericht aus dem unbekannten Land - der Artikel des Wissenschaftsjournalisten - kann und wird die Vorstellung der Leser über lange Zeit prägen. Hinzu kommt, dass viele solcher Themen in den klassischen Medien oft nur ein paar Tage im Rampenlicht stehen und danach häufig monatelang kaum noch wahrgenommen werden. Wenn es sich dann um ein Forschungsgebiet handelt, das gleichzeitig von interessengetriebenen Lobbygruppen welcher Couleur auch immer besetzt wird, zeigt sich, dass Wissenschaftsjournalismus Anforderungen genügen muss, die in dieser Form in vielen anderen Ressorts eher selten sind. Moderner Wissenschaftsjournalismus benötigt Plattformen, die sich dauerhaft mit Zukunftsthemen beschäftigen, doch diese sind selten und häufig für Verlage wirtschaftlich nicht (mehr) attraktiv. Journalisten werden deshalb zunehmend gezwungen, eigene Wege 
zu finden, wenn sie ihre umfangreichen Kenntnisse und Recherchen den Lesern zur Verfügung stellen wollen.

Nach der Lektüre des bisherigen Textes zum Anspruch an den Wissenschaftsjournalismus liegt die Vermutung nahe, dass den Angehörigen dieses Berufsstandes regelmäßig der rote Teppich ausgerollt und ihre Arbeit (auch im wörtlichen Sinne) wertgeschätzt wird. Doch das Gegenteil ist der Fall. Die Honorare für Texte verharren seit Jahren auf niedrigem Niveau. Oft steht die Entlohnung in keinem Verhältnis zum Aufwand einer Recherche und zur Expertise, die manche Kollegen in ihre Texte einbringen. Selbst wenn das Zeilenhonorar attraktiv aussieht, müssen Freiberufler bei ihrer Kalkulation bedenken, dass nicht nur ein Text geliefert werden muss, sondern bei vielen Redaktionen ergänzende Informationen erwartet werden, bis hin zur Aufarbeitung der Quellen für einen Faktencheck, der Zeit erfordert, aber nicht minder wichtig ist als der Text selbst.

Hinzu kommt das Problem des geringer werdenden Platzes: Wenn Verlage sich entscheiden, die Umfänge ihrer Zeitungen und Magazine zu verkleinern, gehört das Ressort Wissenschaft fast immer zu den Verlierern.

Der Wissenschaftsjournalismus in seiner heutigen Form ist in seiner Existenz bedroht. Viele hochqualifizierte Kollegen haben ihre Selbstständigkeit aufgeben müssen und arbeiten in den Abteilungen für Wissenschaftskommunikation, mit denen Universitäten, Unternehmen und Forschungseinrichtungen versuchen, einen größeren Einfluss auf die öffentliche Berichterstattung zu nehmen. Wissenschaftsjournalisten müssen sich eigene Vertriebswege überlegen, um ihr Produkt, das bei den Lesern durchaus Interesse findet, auch finanziell erfolgreich zu präsentieren.

Dabei haben sich die Arbeitsbedingungen für Wissenschaftsjournalisten während der Recherche in manchen Punkten verbessert. Heute ist es einfacher, die Forscher und ihre Arbeit auch außerhalb von Fachjournalen kennenzulernen. Viele Wissenschaftler stellen beispielsweise ihre öffentlichen Vorträge als Video ins Internet. Auch der Anteil der Forscher, die auf eigenen Webseiten oder bei Twitter über ihre Arbeit berichten, ist gewachsen. Die kurzen Tweets erleichtern es, neue Forschungsergebnisse zu finden und Entwicklungen zu verfolgen. Entweder weil die Forscher über eigene Ergebnisse twittern oder weil sie von ihnen als wichtig bewertete Resultate retweeten. Ein Interview via Skype ist für Wissenschaftler Normalität, ebenso wie Hintergrundgespräche, die per Livestream übertragen werden. Beides senkt die Reisekosten von Journalisten. Die Pressearbeit vieler Forschungseinrichtungen hat sich verbessert: Sie liefern mehr begleitendes Material und gute Kommunikationsabteilungen verschweigen auch das Für und Wider ihrer Arbeit nicht. Zudem versorgt das vor wenigen Jahren gegründete Science Media Center die Journalisten zeitnah 
und kompetent mit der Einschätzung anderer Experten zu neuen Publikationen (vgl. den Beitrag von Volker Stollorz). Diesen Service lieferten früher nur die internationalen Wissenschaftsmagazine wie Science und Nature, deren Kommentare oft die Meinung zahlreicher Medien prägten.

In diesen Entwicklungen lauert die Gefahr, dass Wissenschaftsjournalisten ihren Schreibtisch nicht mehr verlassen. Es erscheint bequem, die öffentlich zur Verfügung stehenden Informationen zu sammeln, ein paar wenige Fragen per E-Mail an einen der beteiligten Forscher zu schicken und damit ein Thema abzuarbeiten. Diese Form der Bequemlichkeit wird unterstützt, wenn Verlage in immer geringer werdendem Umfang Reisekosten übernehmen. Diese Arbeitsweise senkt zwar die Kosten des freiberuflichen Journalisten, aber sie schränkt auch die Qualität seiner Arbeit mehr und mehr ein. Der Freiberufler kann nur reagieren. Er wird zum Chronisten dessen, was andere publizieren wollen. Gleichzeitig verpasst er naturgemäß aktuelle Entwicklungen und erfährt diese erst, wenn sie auf Webseiten oder in wissenschaftlichen Fachartikeln auftauchen.

Wissenschaftsjournalisten müssen trotz der schlechten finanziellen Rahmenbedingungen ihren Schreibtisch verlassen. Sie müssen Debatten auf Tagungen verfolgen, Forscher in ihrem Labor besuchen und das direkte Gespräch mit Wissenschaftlern führen. Nur so können sie erfahren, worüber die Akteure wirklich diskutieren und welchen Problemen sie gegenüberstehen. Wirtschaftlich gesehen sind solche Reisen eine Investition in die Zukunft. Sie rechnen sich selten kurzfristig, erweisen sich aber fast immer langfristig als lohnend. Ein Beispiel: Mir wurde die radikale Veränderung der Genforschung durch die neue Genschere CRISPR/Cas deutlich, als mir ein Stammzellforscher erzählte, dass er mehrere Mitarbeiter zu CRISPR/ Cas-Schulungen in die USA geschickt habe. Ich habe danach andere Wissenschaftler befragt, die mir aus ihren Teams ähnliche Dinge schilderten und mir bei dieser Gelegenheit die Stärken und Schwächen des neuen Verfahrens erläuterten. Es waren Plädoyers, aus denen deutlich wurde, dass die Gentechnik vor einer Revolution steht. Zu diesem Zeitpunkt waren Berichte in Medien über diese neue Technologie aber noch ausgesprochen selten.

Tagungen, auf denen solche Gespräche möglich sind, findet man manchmal über die Fachverbände der Wissenschaftler. Gut geeignet sind auch Auftaktveranstaltungen von Sonderforschungsbereichen. Dort ist die Aufbruchstimmung der Wissenschaft am ehesten zu spüren. Veranstaltungen in kleineren Städten sind meistens besser als solche in größeren, weil sie weniger Publikum anlocken, das eher an touristischen Hotspots interessiert ist als an wissenschaftlichen Ergebnissen. Intensive Diskussionsrunden mit vielen Studierenden sind besser als Tagungen, die eher aus Showvorträgen bestehen und bei denen die Referenten schnell verschwinden, statt sich aus- 
zutauschen. Gute Tagungsmanager vermitteln nicht nur Interviews mit Keynote-Speakern, sondern weisen auch auf andere interessante Forscher hin oder auf solche, die „off the records“ über Forschung in anderen Ländern sprechen. Hinzu kommen Gespräche mit Ethikern und Philosophen; Interviews mit Politikern helfen meist nur wenig weiter.

Ähnliches gilt für Laborbesuche. Ich bin immer wieder überrascht, wie viele Forscher bereit sind, die Türen ihrer Institute zu öffnen. Vermutlich bin ich der einzige Wissenschaftsjournalist, der bereits zwei Fabriken zur Herstellung von gentechnisch veränderten Insekten besucht hat. Diese Strategie ermöglicht es, den Themen zu folgen. Es war spannend zu sehen, wie beispielsweise Stammzellforscher über die Jahre ihre Ziele verändert haben. Zunächst haben sie die Züchtung von kompletten Organen angekündigt. Heute stellen sie Organoide als Modelle für die Entwicklung von Krankheiten vor oder lebende menschliche Zellen, die als Test für Medikamente genutzt werden können. Dieses Wissen wiederum verbessert die Qualität der Gespräche mit den Forschern.

Rainer Kurlemann ist promovierter Chemiker und arbeitet seit mehr als 30 Jahren als Journalist. Er war 14 Jahre bei der Rheinischen Post, unter anderem als Chef vom Dienst und als Chefredakteur der Online-Redaktion, und schreibt heute als freiberuflicher Wissenschaftsjournalist für zahlreiche Zeitungen und Magazine. $\mathrm{Zu}$ seinen Schwerpunkten zählen Gentechnik, Stammzellforschung und Umweltschutz. Er ist Autor des Wissenschaftskrimis Der Geranienmann und Mitglied in der Genossenschaft RiffReporter.

Open Access Dieses Kapitel wird unter der Creative Commons Namensnennung 4.0 International Lizenz (http://creativecommons.org/licenses/by/4.0/deed.de) veröffentlicht, welche die Nutzung, Vervielfältigung, Bearbeitung, Verbreitung und Wiedergabe in jeglichem Medium und Format erlaubt, sofern Sie den/die ursprünglichen Autor(en) und die Quelle ordnungsgemäß nennen, einen Link zur Creative Commons Lizenz beifügen und angeben, ob Änderungen vorgenommen wurden.

Die in diesem Kapitel enthaltenen Bilder und sonstiges Drittmaterial unterliegen ebenfalls der genannten Creative Commons Lizenz, sofern sich aus der Abbildungslegende nichts anderes ergibt. Sofern das betreffende Material nicht unter der genannten Creative Commons Lizenz steht und die betreffende Handlung nicht nach gesetzlichen Vorschriften erlaubt ist, ist für die oben aufgeführten Weiterverwendungen des Materials die Einwilligung des jeweiligen Rechteinhabers einzuholen.

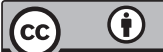

